DOI 10.37882/2223-2982.2021.12.23

\title{
ТИПЫ ДВУСЛОЖНЫХ СИНГАРМОНИЧЕСКИХ ФОНЕТИЧЕСКИХ СТРУКТУР В АЗЕРБАЙДЖАНСКОМ И РУССКОМ ЯЗЫКАХ
}

\section{TYPES OF DISYLLABIC SYNHARMONIC PHONETIC STRUCTURES IN AZERBAIJANI AND RUSSIAN LANGUAGES}

M. Rahtari

Summary: The article provides a detailed linguistic-statistical analysis of the synharmonic phonetic structures of the disyllable lexis of the Azerbaijani and Russian languages. Two types of synharmonic structures S2.1 and S2.2 are distinguished in each of the languages. Structural varieties of each type are established, a comparative analysis of the intralingual productivity of each type of synharmonic structures and their varieties is carried out, as well as an interlingual comparative analysis of the productivity of each type of synharmonic structures and their varieties in the two studied languages.

Keywords: phonetic structural type, synharmonicity, synharmonic/ nonsynharmonic, homogeneous/heterogeneous, agglutinative, flectional, productivity.

\author{
Рахтари Махира Фамиловна \\ аспирант, стариий лаборант, Сумгаитский \\ государственный университет (Азербайджан) \\ arifmeryem@mail.ru
}

Аннотация: В статье дан подробный лингвостатистический анализ сингармонических фонетических структур двусложной лексики азербайджанского и русского языков. Выделены два типа сингармонических структур S2.1 и S2.2 в каждом языке. Установлены структурные разновидности каждого типа, осуществлен сравнительный анализ внутриязыковой продуктивности каждого типа сингармонических структур и их разновидностей, а также межъязыковой сопоставительный анализ продуктивности каждого типа сингармонических структур и их разновидностей в обоих языках.

Ключевые слова: фонетический структурный тип, сингармонизм, сингармонический/несингармонический, однородный/неоднородный, агглютинативный, флективный, продуктивность.
$\mathrm{B}$ статье осуществлена попытка анализа сингармонической фонетической структуры двусложной лексики современных азербайджанского и русского литературных языков с точки зрения нёбной, или тембральной однородности/неоднородности звукосочетаний, которые составляют их фонетический облик. Термин сингармонизм образован слиянием греческих слов syn (вместе) и harmonia (звучание, связь) и означает созвучие, гармония. Ф.Г. Исхаков рассматривает сингармонизм как гармонию гласных в составе слова и пишет: «Сущность небного (палатального) сингармонизма заключается в гармонии гласных, входящих в состав слова, по "твердости" и “мягкости"» [1, с. 89]. Однако следует учесть, что гармония звуков речи - это не только гармония гласных в составе слова, но и гармония между гласными и окружающими их согласными в составе звукосочетания и в составе слова. В этом смысле А.А. Реформатский справедливо подчеркивал, что сингармонизм распространяется на всё слово в целом; это явление представляет собой суперсегментную «силу», «накладку» - и не на отдельные сегменты, а на целое [7, с. 104].

Мы употребляем термин сингармонизм вслед за Р.С. Мамедовым для обозначения однородности/неоднородности гласных и согласных по признаку их твердости-мягкости в составе вокально-консонантного звукосочетания и в составе слова в соответствии с нёбной гармонией (тембральный, или палатальный сингармонизм) [2, с. 58; 3, с. 7]. Сингармонизм обеспечивает однородность гласных и согласных фонем по признаку их твёрдости или мягкости. Например, гласные и согласные в таких словах, как вода, дети в русском языке и qanun (закон), ӥrәk (сердие) в азербайджанском являются однородными, то есть сингармоничными по признаку их твердости (вода, qanun) и мягкости (дети, ürək). Сингармонические слова обознаются символом $\mathbf{S}$, а несингармонические - символом $\overline{\mathbf{S}}$. Цифра $\mathbf{2}$ после $\mathbf{S}$ и $\overline{\mathbf{S}}$ обозначает количество вокально-консонантных звукосочетаний, или количество слогов в слове: Таким образом, S2 означает - сингармоническое двусложное слово, а $\mathbf{\mathbf { 5 }}$ - несингармоническое двусложное слово [3, c. 13, 104-109].

Двусложные слова для анализа их сингармонической фонетической структуры отобраны нами из орфографических словарей современного азербайджанского и современного русского литературных языков $[5 ; 6]$. Из 110563 слов, включенных в состав словаря азербайджанского языка, двусложными являются 11216 слов, или более $10 \%$ всей лексики данного языка. Из них сингармонической структурой обладает 8291 слово, что составляет 73,9\% всей двусложной лексики азербайджанского языка, а несингармонической структурой - 2925 слов, что составляет $26,1 \%$ всей двусложной лексики азербайджанского языка. Таким образом, количественное соотношение сингармонической и несингармонической 
двусложной лексики (S2 и $\mathbf{S} \mathbf{2})$ азербайджанского языка составляет пропорцию 3:1. Другими словами, каждое четвертое двусложное слово азербайджанского языка

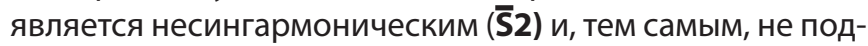
чиняется такому фундаментальному фонетическому закону азербайджанского языка, как закон гармонии звуков, или сингармонизм.

Из 100000 слов, включенных в состав словаря русского языка, двусложными являются всего 9426 слов, что составляет немногим более $9 \%$ всей лексики русского языка. Из них сингармонической структурой обладает 2201 слово, что составляет 23,4\% всей двусложной лексики русского языка, а несингармонической структурой - 7225 слов, что составляет 76,6\% всей двусложной лексики русского языка. Количественное соотношение сингармонической и несингармонической двусложной лексики (S2 и $\mathbf{\mathbf { S }}$ ) русского языка также составляет пропорцию 3:1, однако с той принципиальной разницей, что сингармонические и несингармонические слова в

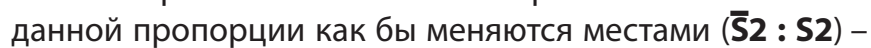
теперь каждое четвертое двусложное слово русского языка является сингармоническим, типологически совпадая с двусложными сингармоническими структурами азербайджанского языка.

Таким образом, сингармонические (S2) и несингар-

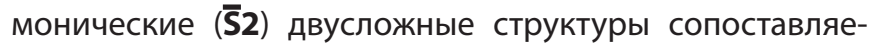
мых языков, формально составляя почти одинаковую пропорцию, выражают, тем не менее, принципиальные структурные расхождения двух неродственных, разно системных языков: азербайджанского как агглютинативного и русского как фузионного. Сказанное о соотношениях $\mathbf{S} 2$ и $\mathbf{S} \mathbf{2}$ двусложных слов обобщенно представлено ниже в Таблице 1.

Двусложные слова с сингармонической фонетической структурой (S2) делятся на два типа: S2.1 и S2.2.

1. S2.1 - двусложное слово сингармонической фонетической структуры первого типа с вокальным компонентом «однородный гласный непереднего ряда». Этот тип реализован различными комбинациями, типа a+ta, at+tat, tat+tat, ta+ta, tat+tatt u m.n. звукосочетаний, образованных твёрдыми гласными (a) и твёрдыми согласными ( $t, t t, t t t .$.$) .$ Если обозначить эти сочетания гласных и согласных звуков символом С1, то формула твёрдого сингармонизма в двусложных словах будет определена следующим образом: С1 + С1 [3, с. 13-14]

2. $\mathbf{S 2 . 2}$ - двусложное слово сингармонической фонетической структуры второго типа с вокальным компонентом «однородный гласный переднего ряда». Этот тип также реализован различными комбинациями звукосочетаний, состоящими из мягкого гласного (i) и мягкого согласного ( $\left.t^{\prime}\right)$ или мягких согласных $\left(t^{\prime} t^{\prime}, t^{\prime} t^{\prime} t^{\prime}\right)$. Сочетания данного типа обозначим символом С2. Таким образом, С2 второй тип сингармонического сочетания, состоящий из комбинаций мягкого гласного и мягкого согласного, типа $\boldsymbol{i}+\boldsymbol{t}^{\prime} \boldsymbol{i} \boldsymbol{t}^{\prime}, \boldsymbol{i t}^{\prime}+\boldsymbol{t}^{\prime} \boldsymbol{i}, \boldsymbol{t}^{\prime} \boldsymbol{i}+\boldsymbol{t}^{\prime} \boldsymbol{i}, \boldsymbol{t}^{\prime} \boldsymbol{i}+\boldsymbol{t}^{\prime} \boldsymbol{i t} \boldsymbol{t}^{\prime} \boldsymbol{t}^{\prime} \boldsymbol{i t}^{\prime}$ + t'it', it' + t'it' и т. п., который условно можно назвать мягким сингармонизмом. Формула мягкого сингармонизма в двусложных словах будет обозначена следующим образом: С2 + C2.

\section{$\triangle$ вусложные сингармонические фонетические структурные разновиАности азербайАканского языка}

В Таблице 2 представлены лингвостатистические данные о двусложных сингармонических фонетических структурных разновидностях азербайджанского языка в порядке их количественного убывания.

Лингвостатистический комментарий к Таблице 2:

1. В азербайджанском языке имеется всего 8291 слово двусложной сингармонической структуры. Из них 4190 слов, или 50,5\% всей двусложной сингармонической лексики азербайджанского языка реализованы 47 разновидностями фонетического структурного типа S2.1 (C1+C1). Остальное 4101 слово реализовано 39 разновидностями фонетического структурного типа S2.2 (C2+C2), что составляет 49,5\% двусложной сингармонической двусложной лексики этого языка.

2. Средняя функциональная нагрузка (далее: продуктивность) одной фонетической структурной разновидности в группе структурного типа S2.1 составляет чуть более 89 слов. В группе структурного типа S2.2 этот показатель заметно вышечуть более 105 слов.

3. Минимальная продуктивность одной фонетической структурной разновидности составляет 1 слово, максимальная: в группе структурного типа

Таблица 1.

Соотношение сингармонических и несингармонических двусложных слов в азербайджанском и русском языках.

\begin{tabular}{|c|c|c|c|c|c|c|}
\hline \multirow{2}{*}{ Языки } & \multirow{2}{*}{ Всего слов } & \multicolumn{2}{|c|}{ сингармонических (S2) } & \multicolumn{2}{|c|}{ несингармонических ( $\bar{S} 2)$} & \multirow{2}{*}{ Пропорции S2: $\bar{S} 2$} \\
\hline & & кол-во & B $\% \%-x$ & кол-во & B $\% \%-x$ & \\
\hline Азербайджанский & 11216 & 8291 & $73,9 \%$ & 2925 & $26.1 \%$ & $3: 1$ \\
\hline Русский & 9426 & 2201 & $23,4 \%$ & 7225 & $76.6 \%$ & $1: 3$ \\
\hline
\end{tabular}


Таблица 2.

Двусложные сингармонические фонетические структурные разновидности азербайджанского языка в порядке их количественного убывания

\begin{tabular}{|c|c|c|c|c|c|c|c|}
\hline \multicolumn{4}{|c|}{$\mathrm{S} 2.1(\mathrm{C} 1+\mathrm{C} 1)$} & \multicolumn{4}{|c|}{$\mathrm{S} 2.2(\mathrm{C} 2+\mathrm{C} 2)$} \\
\hline Тип & Пример & Кол-во & $\%$ & Тип & Пример & Кол-во & $\%$ \\
\hline 1. tattat & baqqal & 1271 & 30.33 & 1. t'it't'tit' $^{\prime}$ & hörmət & 1117 & 27.23 \\
\hline 2. tatat & qanun & 894 & 21.33 & 2. t'it'it' $^{\prime}$ & hәуәt & 864 & 21.06 \\
\hline 3. tatta & qarğa & 642 & 15.32 & 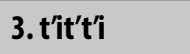 & kəllə & 685 & 16.70 \\
\hline & & 2807 & $70 \%$ & & & 2666 & $65 \%$ \\
\hline 4. tata & buta & 258 & 6.15 & 4. t'it'i & cücә & 268 & 6.53 \\
\hline 5. attat & anlam & 221 & 5.27 & 5. it't'it' & әzgil & 215 & 5.24 \\
\hline 6. atat & ocaq & 149 & 3.55 & 6. it'it' & ürək & 138 & 3.36 \\
\hline 7. tatttat & qalstuk & 114 & 2.72 & 7. t'it't't'it' & pinqvin & 165 & 4.02 \\
\hline & & 742 & $18 \%$ & 8. it't'i & iydə & 105 & 2.56 \\
\hline & & & & 9. $t^{\prime} \mathbf{t}^{\prime} \mathrm{t}^{\prime} \mathrm{t}^{\prime} \mathbf{i}$ & türkcə & 145 & 3.53 \\
\hline 8. atta & alça & 83 & 1.98 & & & 1036 & $25 \%$ \\
\hline 9. tattta & tundra & 72 & 1.71 & & & & \\
\hline 10. ttattat & fransız & 61 & 1.45 & 10. $t^{\prime} t^{\prime} i^{\prime} t^{\prime} t^{\prime} t^{\prime}$ & printer & 28 & 0.68 \\
\hline 11. ttatta & spazma & 54 & 1.28 & 11. t't'it't'i & qrifli & 8 & 0.19 \\
\hline 12. tattatt & komfort & 52 & 1.24 & 12. $t^{\prime} t^{\prime} t^{\prime} t^{\prime} t^{\prime} t^{\prime}$ & xərçəng & 83 & 2.02 \\
\hline 13. tatatt & balans & 51 & 1.21 & 13. t'it'it't't' & kükürd & 77 & 1.87 \\
\hline 14. ttatat & pluton & 45 & 1.07 & 14. $t^{\prime} t^{\prime} i t^{\prime} i t^{\prime}$ & steril & 23 & 0.56 \\
\hline 15. tatttatt & kontrast & 41 & 0.97 & 15. 'it't't't'tit't' & linqvist & 31 & 0.75 \\
\hline 16. ata & ağı & 30 & 0.71 & 16. it'i & ütü & 22 & 0.53 \\
\hline 17. taat & sual & 16 & 0.38 & 17. t'iit' & rəis & 8 & 0.19 \\
\hline 18. attatt & avqust & 16 & 0.38 & 18. it't'tit't' & ellips & 9 & 0.21 \\
\hline 19. ttata & plata & 14 & 0.33 & 19. t't'it'i & plitə & 2 & 0.04 \\
\hline 20. atatt & axund & 13 & 0.31 & 20. it'it't't & әhәng & 8 & 0.19 \\
\hline 21. ttatttat & smartfon & 12 & 0.28 & 21.t't'tit't't'it' & prinslik & 6 & 0.14 \\
\hline 22. atttat & arslan & 10 & 0.23 & 22. it't't'tit' & emblem & 23 & 0.56 \\
\hline 23. ttattatt & standart & 9 & 0.21 & 23. t't' $^{\prime} \mathrm{it}^{\prime} \mathrm{t}^{\prime} \mathrm{it}^{\prime} \mathrm{t}^{\prime}$ & pressinq & 6 & 0.14 \\
\hline 24. ttattta & tromblu & 9 & 0.21 & 24. $t^{\prime} t^{\prime} i^{\prime} t^{\prime} t^{\prime} i$ & spirtli & 8 & 0.19 \\
\hline 25. tattttat & substrat & 8 & 0.19 & 25.t'it't't't'tit' & centlmen & 5 & 0.12 \\
\hline 26. attta & astma & 7 & 0.16 & 26. it't't'i & ölçmə & 16 & 0.39 \\
\hline 27. taa & dua & 5 & 0.11 & 27. t'ii & şiə & 2 & 0.04 \\
\hline 28. tattttatt & kart-blanş & 3 & 0.07 & - & - & - & - \\
\hline 29. ttatatt & florans & 3 & 0.07 & 28. $t^{\prime} t^{\prime} i t^{\prime} i t^{\prime} t^{\prime}$ & stilist & 7 & 0.17 \\
\hline 30. at & aut & 2 & 0.04 & - & - & - & - \\
\hline 31. taatt & raund & 2 & 0.04 & 29. $t^{\prime} i^{\prime} t^{\prime} t^{\prime}$ & teizm & 7 & 0.17 \\
\hline 32. tatattt & kadastr & 2 & 0.04 & 30. $t^{\prime} \mathrm{it}^{\prime} \mathrm{it}^{\prime} \mathrm{t}^{\prime} \mathrm{t}^{\prime}$ & reyestr & 4 & 0.09 \\
\hline
\end{tabular}




\begin{tabular}{|l|c|c|c|}
\hline \multicolumn{5}{|c|}{ S2.1 (C1+C1) } \\
\hline Tип & Пример & Кол-во & $\%$ \\
\hline 33. ttaat & kloun & 2 & 0.04 \\
\hline 34. ttatttatt & krossvord & 2 & 0.04 \\
\hline 35. tttatta & knyajna & 2 & 0.04 \\
\hline 36. tttattat & struktur & 2 & 0.04 \\
\hline 37. attattt & ansambl & 2 & 0.04 \\
\hline 38. atttatt & oktyabr & 2 & 0.04 \\
\hline 39. attttat & abstrat & 1 & 0.02 \\
\hline 40. attttatt & abstrakt & 1 & 0.02 \\
\hline 41. tatttta & marksç & 1 & 0.02 \\
\hline 42. ttaa & myao(-lar) & 1 & 0.02 \\
\hline 43. ttatattt & skafandr & 1 & 0.02 \\
\hline 44. tttata & slyapa & 1 & 0.02 \\
\hline 45. tttatatt & strofant & 1 & 0.02 \\
\hline 46. ttattttat & vzvod-vzvod & 1 & 0.02 \\
\hline 47. ttttata & mstyora & 1 & 0.02 \\
\hline - & - & - & - \\
\hline Bcezo: 47 вudoв & & - & - \\
\hline
\end{tabular}

1. S2.1 - 1271 слово, в группе структурного типа S2.2 - 1117 слов. Процентное соотношение - 53\%: 47 \% показывает наибольшую продуктивность структурной разновидности tattat. На втором месте по данному показателю находится структурная разновидность t'it't'it'.

2. Первые 3 разновидности в обеих группах структурного типа являются наиболее продуктивными: в группе структурного типа S2.1 на их долю приходится 2807 слов, или 70\% всех слов данного структурного типа; в группе структурного типа S2.2 этот показатель составляет 2666 слов или 65\% всех слов этого структурного типа.

3. Вторую продуктивную группу составляют в группе структурного типа S2.1 4 разновидности (на их долю приходится 742 слова, или 18\% всех слов этого структурного типа), а в группе структурного типа S2.2 - 6 разновидностей (на их долю приходится 1036 слов или 25\% всех слов этого структурного типа).

4. Таким образом, в группе структурного типа S2.1 всего 7 разновидностей реализуют 3549 слов или 75 \% всех слов данного структурного типа; а в группе структурного типа S2.2 - всего 9 разновидностей реализуют 3702 слова или 90\% всех слов данного структурного типа.

\begin{tabular}{|c|c|c|c|}
\hline \multicolumn{4}{|c|}{$\mathrm{S} 2.2(\mathrm{C} 2+\mathrm{C} 2)$} \\
\hline Тип & Пример & Кол-во & $\%$ \\
\hline - & - & - & - \\
\hline 31.t't't'it't't'tit't't' & bremsberq & 1 & 0.02 \\
\hline 32. $t^{\prime} t^{\prime} t^{\prime} i t^{\prime} t^{\prime} i$ & ştrixli & 1 & 0.02 \\
\hline 33.t't't't'it't'it' & sprinter & 3 & 0.07 \\
\hline - & - & - & - \\
\hline 34. it't't't'tit't' & ins-cins & 4 & 0.09 \\
\hline 35. it't't't't'tit' & endşpil & 1 & 0.02 \\
\hline - & - & - & - \\
\hline 36. $t^{\prime} \mathrm{t}^{\prime} \mathrm{t}^{\prime} \mathrm{t}^{\prime} \mathrm{t}^{\prime} \mathrm{i}$ & tembrli & 3 & 0.07 \\
\hline- & - & - & - \\
\hline 37.t't'tit'it't't't' & trimestr & 1 & 0.02 \\
\hline- & - & - & - \\
\hline- & - & - & - \\
\hline- & - & - & - \\
\hline- & - & - & - \\
\hline 38. $t^{\prime} t^{\prime} i^{\prime} t^{\prime} t^{\prime}$ & flüens & 1 & 0.02 \\
\hline 39. $t^{\prime} i t^{\prime} t^{\prime} t^{\prime} i t^{\prime} t^{\prime} t^{\prime}$ & leyttembr & 1 & 0.02 \\
\hline \multicolumn{2}{|l|}{ Всего: 39 видов } & 4101 слов & $100 \%$ \\
\hline
\end{tabular}

5. Третью группу по продуктивности составляют в группе структурного типа S2.1 15 разновидностей (на их долю приходится 742 слова или 18\% всех слов этого структурного типа), а в группе структурного типа S2.2 - 8 разновидностей (на их долю приходится 303 слова или чуть более 7\% всех слов этого структурного типа).

6. Последнюю группу по продуктивности составляют в группе структурного типа S2.1 - 25 разновидностей (на их долю приходится всего лишь 71 слово, или менее 3\% всех слов этого структурного типа), а в группе структурного типа S2.2 - 17 разновидностей (их доля - 88 слов или $2 \%$ всех слов этого структурного типа).

\section{$\triangle$ вусложные сингармонические фонетические структурные разновидности русского языка}

Лингвостатистические данные о двусложных сингармонических фонетических структурных разновидностях русского языка в порядке их количественного убывания представлены в Табличе 3.

\section{Лингвостатистический комментарий к Таблице 3.}

1. В русском языке имеется всего 2201 слово двусложной сингармонической структуры. Из них 1991 
Таблица 3.

Двусложные сингармонические фонетические структурные разновидности русского языка в порядке их количественного убывания

\begin{tabular}{|c|c|c|c|c|c|c|c|}
\hline \multicolumn{4}{|c|}{$\mathrm{S} 2.1(\mathrm{C} 1+\mathrm{C} 1)$} & \multicolumn{4}{|c|}{$\mathrm{S} 2.2(\mathrm{C} 2+\mathrm{C} 2)$} \\
\hline Тип & Пример & Кол-во & $\%$ & Тип & Пример & Кол-во & $\%$ \\
\hline 1. tatat & донор & 420 & 21.09 & 1. t'it'it' & сидеть & 72 & 34.28 \\
\hline 2. tattat & ландыш & 299 & 15.01 & 2. t'it't'it' & винтить & 27 & 12.85 \\
\hline 3. tatta & кошка & 294 & 14.76 & 3. t'it't'i & серьги & 23 & 10.95 \\
\hline 4. tata & зона & 162 & 8.13 & 4. t'it'i & филе & 29 & 13.80 \\
\hline 5. ttatta & крышка & 119 & 5.97 & 5. $t^{\prime} t^{\prime} i^{\prime} t^{\prime} i$ & свести & 5 & 2.38 \\
\hline 6. ttata & проба & 108 & 5.42 & 6. t' t'it'i & стихи & 2 & 0.95 \\
\hline 7. attat & образ & 93 & 4.67 & 7. it't'it' & избить & 9 & 4.28 \\
\hline 8. ttatat & бросок & 90 & 4.52 & 8. $t^{\prime} t^{\prime} i t^{\prime} i t^{\prime}$ & светить & 25 & 11.90 \\
\hline 9. atat & овал & 59 & 2.96 & 9. it'it' & иметь & 2 & 0.95 \\
\hline 10. tatttat & завтрак & 38 & 1.90 & - & - & - & - \\
\hline 11. ttattat & флагман & 36 & 1.80 & 10.t't'tit't'it' & сместись & 9 & 4.28 \\
\hline 12. tatatt & курорт & 35 & 1.75 & 11.t'it'it't't' & нечисть & 2 & 0.95 \\
\hline 13. tattatt & сарказм & 35 & 1.75 & - & - & - & - \\
\hline 14. atta & ОКНO & 24 & 1.20 & 12. it't'i & uдmu & 3 & 1.42 \\
\hline 15. tattta & завтра & 20 & 1.00 & - & - & - & - \\
\hline 16. tttatta & вкладка & 18 & 0.90 & - & - & - & - \\
\hline 17. ata & $y x a$ & 14 & 0.70 & 13. it'i & или & 1 & 0.47 \\
\hline 18. tttata & справа & 14 & 0.70 & - & - & - & - \\
\hline 19. atatt & обыск & 12 & 0.60 & - & - & - & - \\
\hline 20. atttat & omcmyn & 10 & 0.50 & - & - & - & - \\
\hline 21. attatt & абсурд & 9 & 0.45 & 14.it't'tit't' & известь & 1 & 0.47 \\
\hline 22. taat & баул & 9 & 0.45 & - & - & - & - \\
\hline 23.ttattatt & сканворд & 8 & 0.40 & - & - & - & - \\
\hline 24. tatttta & чарство & 7 & 0.35 & - & - & - & - \\
\hline 25.ttatttat & планктон & 7 & 0.35 & - & - & - & - \\
\hline 26.ttatttta & братство & 6 & 0.30 & - & - & - & - \\
\hline 27. attta & acmpa & 5 & 0.25 & - & - & - & - \\
\hline 28. taa & moy & 5 & 0.25 & - & - & - & - \\
\hline 29.tattttat & субстрат & 5 & 0.25 & - & - & - & - \\
\hline 30. ttaat & скауm & 4 & 0.20 & - & - & - & - \\
\hline 31.tatttatt & ландшафт & 4 & 0.20 & - & - & - & - \\
\hline 32. ttatatt & продукт & 4 & 0.20 & - & - & - & - \\
\hline 33.ttatttatt & тра́нспорт & 3 & 0.15 & - & - & - & - \\
\hline 34. atttatt & антракт & 2 & 0.10 & - & - & - & - \\
\hline 35. tttatat & вкладыш & 2 & 0.10 & - & - & - & - \\
\hline
\end{tabular}




\begin{tabular}{|c|c|c|c|}
\hline \multicolumn{4}{|c|}{$\mathrm{S} 2.1(\mathrm{C} 1+\mathrm{C} 1)$} \\
\hline Тип & Пример & Кол-во & $\%$ \\
\hline 36. aat & аул & 1 & 0.05 \\
\hline 37. attttat & андшиууг & 1 & 0.05 \\
\hline 38. ttaa & фpay & 1 & 0.05 \\
\hline 39. taatt & раунд & 1 & 0.05 \\
\hline 40. ttattta & столбцы & 1 & 0.05 \\
\hline 41. tttaat & cmpayc & 1 & 0.05 \\
\hline 42.tattttat & танцкласс & 1 & 0.05 \\
\hline 43. tatattt & кадастр & 1 & 0.05 \\
\hline 44.tttattat & uтрафбат & 1 & 0.05 \\
\hline 45.ttattttat & спортклуб & 1 & 0.05 \\
\hline 46.ttatattt & скафандр & 1 & 0.05 \\
\hline \multicolumn{2}{|c|}{ Всего: 46 видов } & 1991 слов & $100 \%$ \\
\hline
\end{tabular}

слово или более 90\% всей двусложной сингармонической лексики руссколго языка реализованы 46 разновидностями фонетического структурного типа S2.1 (C1+C1). Остальные 210 слов реализованы 14 разновидностями фонетического структурного типа S2.2 (C2+C2), что составляет менее 10\% всей двусложной сингармонической лексики русского языка.

2. Средняя продуктивность одной фонетической структурной разновидности в группе структурного типа S2.1 составляет чуть более 43 слов. В группе структурного типа S2.2 этот показатель почти 3 раза ниже - всего 15 слов.

3. Минимальная фактическая продуктивность одной фонетической структурной разновидности составляет 1 слово, максимальная: в группе структурного типа S2.1 - 420 слов, в группе структурного типа S2.2 - 72 слова. Процентное соотношение - 85\%: $15 \%$ показывает наибольшую продуктивность структурной разновидности типа S2.1 - tatat. Ha втором месте по данному показателю находится структурная разновидность типа S2.2 - t'it'it'.

4. Первые 6 разновидностей в группе структурного типа S2.1 являются наиболее продуктивными: на их долю приходится 1402 слова, или более 70\% всех слов данного структурного типа. Средняя фактическая продуктивность одной разновидности в этой группе составляет 234 слова. В группе структурного типа S2.2 нет структурных разновидностей с такой продуктивностью.

5. На долю следующих 8 разновидностей в группе структурного типа S2.1 приходится 410 слов или почти 21\% всех слов этого структурного типа. Средняя фактическая продуктивность в этой группе разновидностей составляет 51 слово. В

\begin{tabular}{|c|c|c|c|}
\hline \multicolumn{5}{|c|}{ S2.2 (C2+C2) } \\
\hline Тип & Пример & Кол-во & $\%$ \\
\hline- & - & - & - \\
\hline- & - & - & - \\
\hline- & - & - & - \\
\hline- & - & - & - \\
\hline- & - & - & - \\
\hline- & - & - & - \\
\hline- & - & - & - \\
\hline- & - & - & - \\
\hline- & - & - & - \\
\hline- & - & - & - \\
\hline Bсего:14 видов & 210 слов & $100 \%$ \\
\hline
\end{tabular}

группе структурного типа S2.2 лишь 1 разновидность имеет сопоставимую продуктивность - это структурная разновидность t'it'it', на долю которого приходится 72 слова или 34\% всех слов данного структурного типа.

6. Наиболее продуктивными в группе структурного типа S2.2 являются 5 разновидностей (1-4 и 8-я разновидности в Таблице), на долю которых приходится всего 176 слов или почти 35\% всех слов данного структурного типа. Средняя фактическая продуктивность в этой группе разновидностей составляет 35 слов.

7. Следующую группу составляют структурные разновидности с продуктивностью от 1 до 20 слов. На долю 32 разновидностей в группе структурного типа S2.1 приходится 169 слов или 8,5\% всех слов этого структурного типа. Средняя фактическая продуктивность в этой группе разновидностей составляет чуть более 5 слов. На долю 9 разновидностей в группе структурного типа S2.2 приходится 34 слова или около 16\% всех слов этого структурного типа. Средняя фактическая продуктивность в этой группе разновидностей составляет менее 4 слов.

\section{Основные выво $ы$}

8. Как в азербайджанском, так и в русском языке более употребительны фонетические структурные разновидности сингармонического типа S2.1: в азербайджанском языке таких разновидностей 47, в русском - 46.

9. Средний показатель продуктивности одной структурной разновидности в группе структурного типа S2.1 в азербайджанском языке составляет 89 слов, в русском - 43 слова. Межъязыковое со 
1. отношение продуктивности в этой группе разновидностей составляет примерно 2:1.

2. Фонетических структурных разновидностей сингармонического типа $\mathbf{5 2 . 2}$ в азербайджанском языке - 39, в русском - 14.

3. Средний показатель продуктивности одной структурной разновидности в группе структурного типа S2.2 в азербайджанском языке составляет
105 слов, в русском - 15 слов. Межъязыковое соотношение продуктивности в этой группе разновидностей составляет примерно 7:1.

4. Соотношение количества слов по структурным типам в азербайджанском языке примерно равное (4190 в группе S2.1 и 4101 - в группе S2.2), а в русском - соотношение 9,5:1 (1991 слово в группе S2.1 и 210 слов - в группе S2.2).

\section{ЛИТЕРАТУРА}

1. Исхаков Ф.Г. Гармония гласных в тюркских языках. Исследование по сравнительной грамматике тюркских языков. Ч. І: Фонетика. М.: Изд-во АН СССР, 1955. С. 122-159.

2. Мамедов Р.С. Вокально-консонантная синтагматика в русском и азербайджанском языках (контрастивно-типологическое системное исследование). Баку: «Мутарджим», 2006. 308 с.

3. Мамедов Р.С. Фонетическая структура слова русского и азербайджанского языков (контрастивно-типологическое системное исследование). Баку: «Мутарджим», 2007. $240 \mathrm{c}$.

4. Новый орфографический словарь русского языка / Составитель И.А. Кузьмина. М.: 000 «Дом славянской книги», 2014. 960 с.

5. Rahtari M.F. Azərbaycan dilinin ikihecalı sözlərinin sinharmonik fonetik struktur növləri // Elmi xəbərlər. Sosial və humanitar elmlər bölməsi. 2019. Cild 15. № 4. S. 12-17.

6. Rahtari M.F. Rus dilində ikihecalı sinharmonik sözlərin fonetik struktur növləri, Sumqayıt Dövlət Universiteti // Elmi xəbərlər. Sosial və humanitar elmlər bölməsi. 2019. Cild 15. № 2. S. 18-24.

7. Реформатский А.А. Сингармонизм как проблема фонологии и общей лингвистики // Тюркологические исследования. Фрунзе: Илим, 1970, С. $101-105$.

8. Орфографический словарь азербайджанского языка. 6-е изд. Баку: «ङərq-Qәrb», 2013.840 c.

\section{() Рахтари Махира Фамиловна (arifmeryem@mail.ru). \\ Журнал «Современная наука: актуальные проблемы теории и практики»}

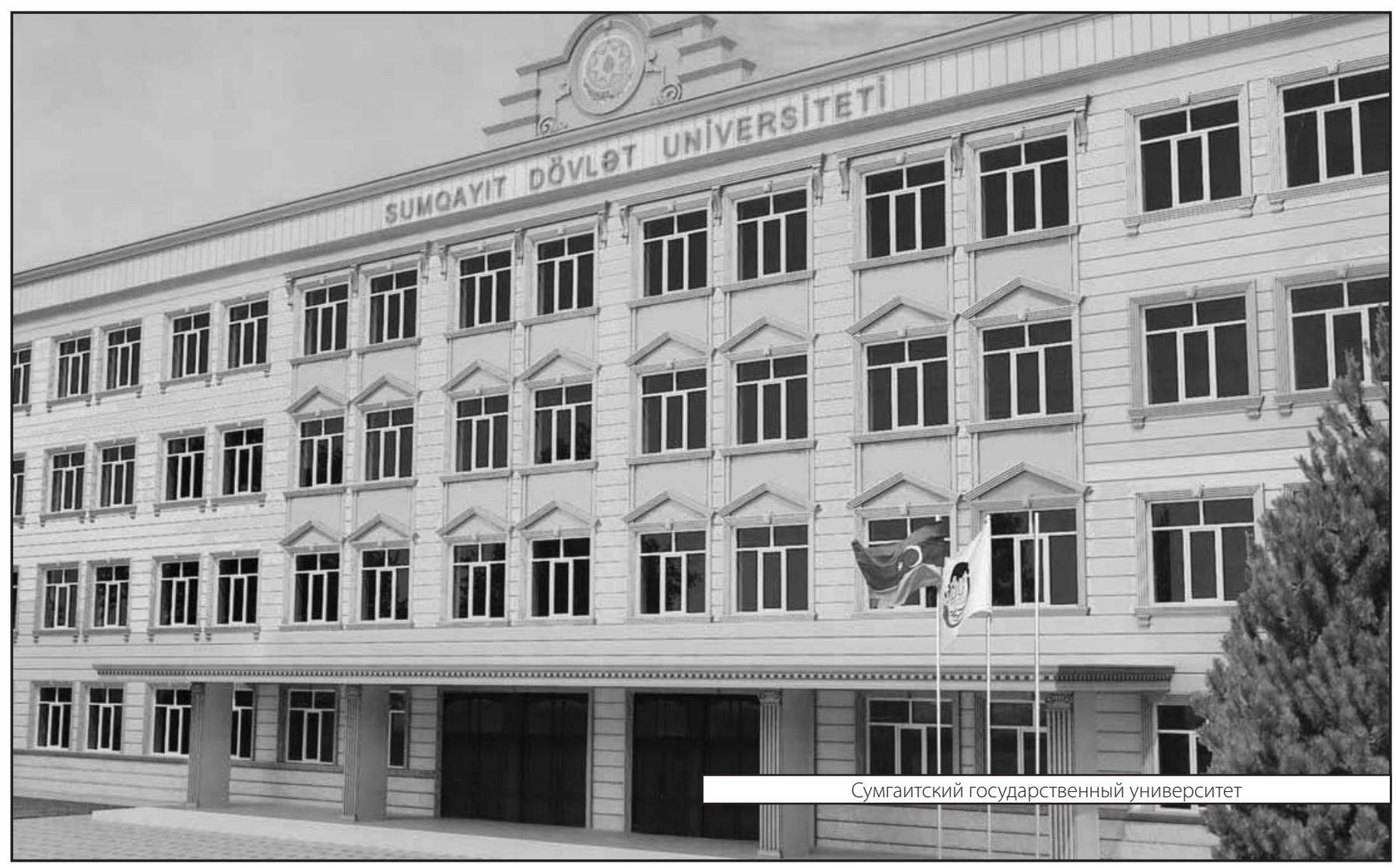

